\title{
Supporting information: Velocity Map Imaging VUV Angle-Resolved Photoemission on Isolated Nanosystems: Case of Gold Nanoparticles
}

\author{
D.K. Božanić1,2,* , G. A. Garcia ${ }^{1}$, O. Sublemontier ${ }^{3}$, J. Pajovic ${ }^{4}$, V. Djoković ${ }^{2}$, L. Nahon ${ }^{1, *}$ \\ ${ }^{1}$ DESIRS beamline, Synchrotron SOLEIL, I'Orme des Merisiers, St. Aubin, BP48, 91192 Gif sur Yvette \\ Cedex, France \\ ${ }^{2}$ Vinča Institute of Nuclear Sciences, University of Belgrade, P.O. Box 522, 11001 Belgrade, Serbia \\ ${ }^{3}$ CEA - Université Paris-Saclay, IRAMIS-NIMBE- Laboratoire Edifices Nanométriques, Bat. 522, 91191 Gif \\ Sur Yvette Cedex, France \\ ${ }^{4}$ Faculty of Physics, University of Belgrade, P.O. Box 368. 11001 Belgrade, Serbia
}

*corresponding authors: bozanic@vin.bg.ac.rs; laurent.nahon@synchrotron-soleil.fr 

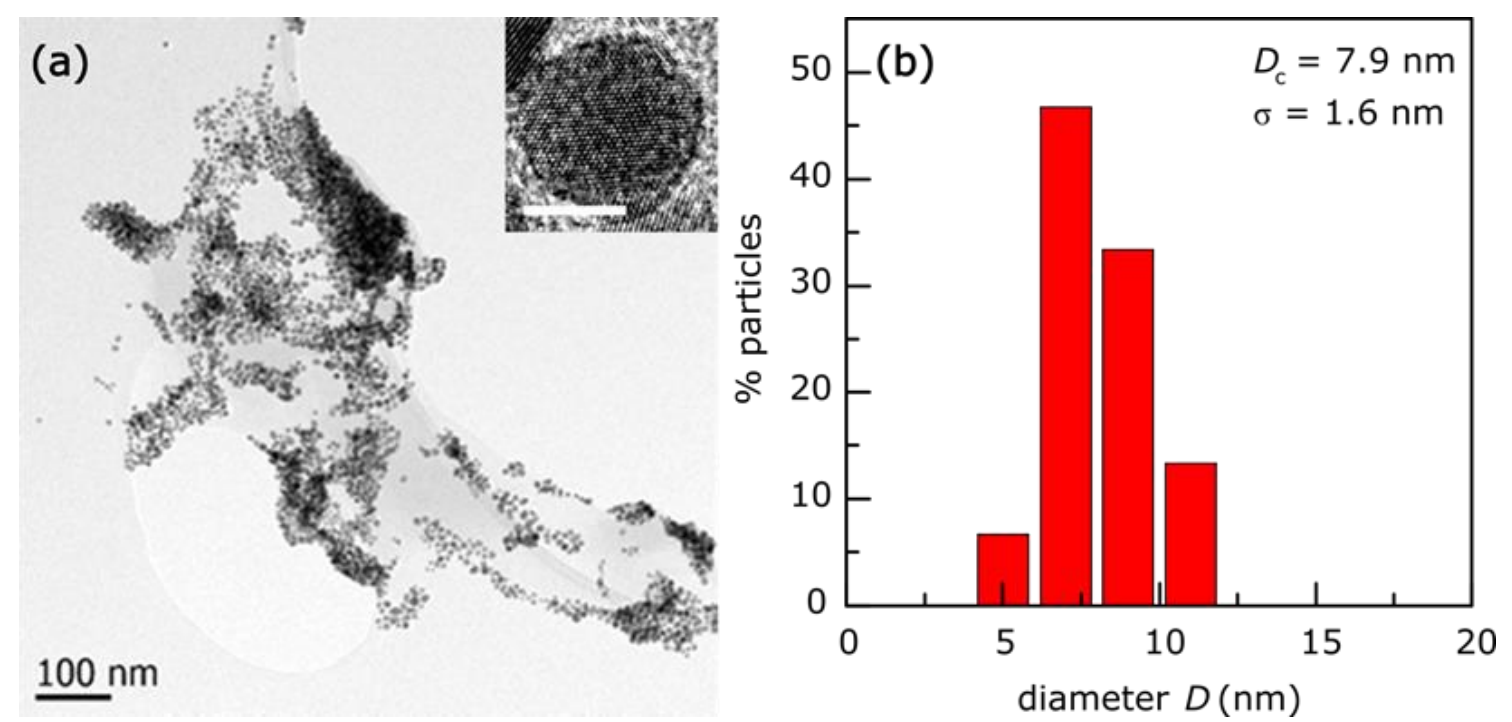

Figure S1. (a) TEM image and (b) the corresponding size distribution of bare gold nanoparticles (AuNP-0). The inset to figure (a) shows a high-resolution image of an individual particle (bar=5 $\mathrm{nm}$ ). The sample was investigated by a TECNAI F30 instrument operating at $300 \mathrm{kV}$ and a point-to-point resolution of $0.205 \mathrm{~nm}$.

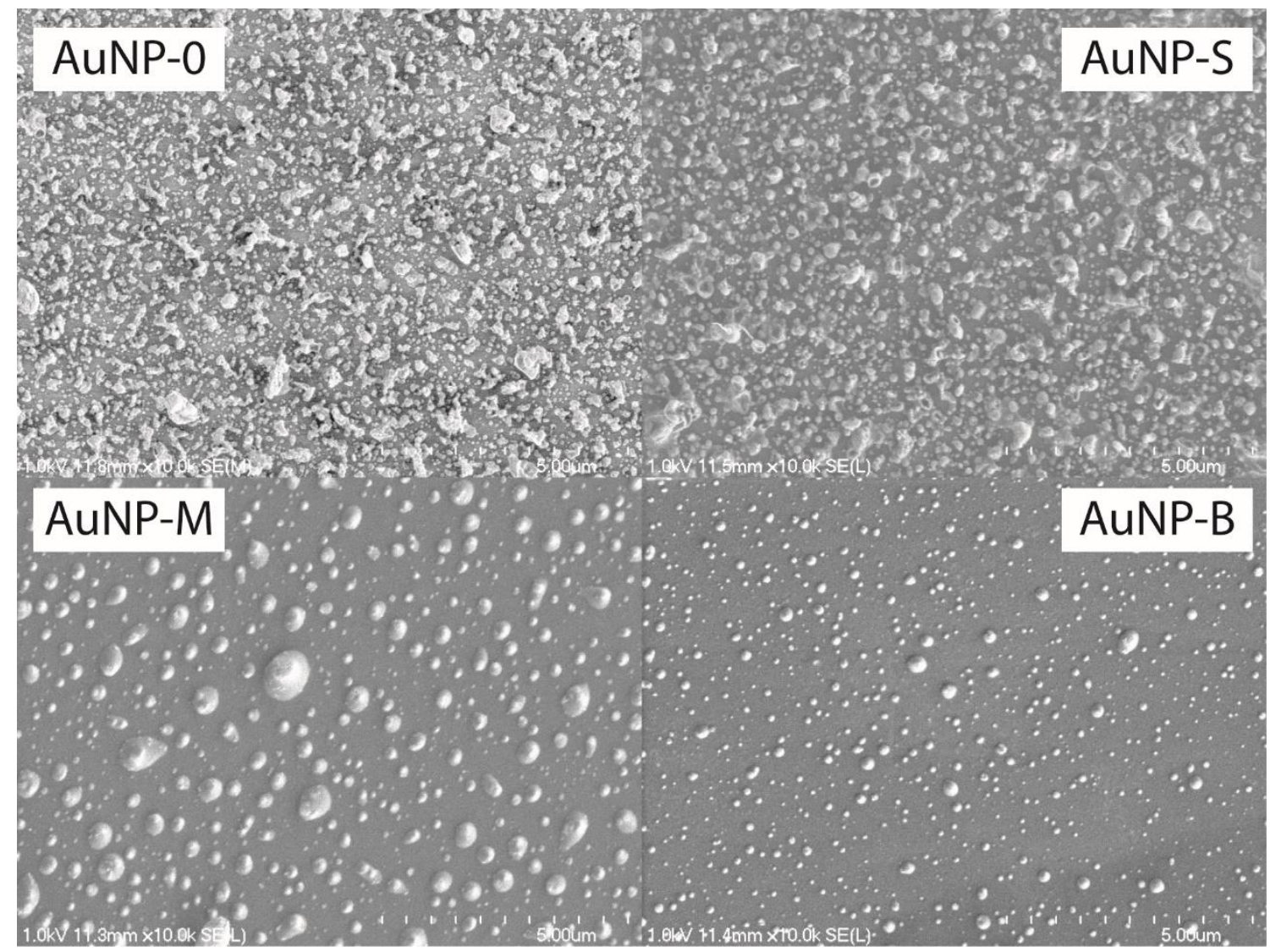

Figure S2. SEM images of aerosol samples deposited onto a conductive substrate. 


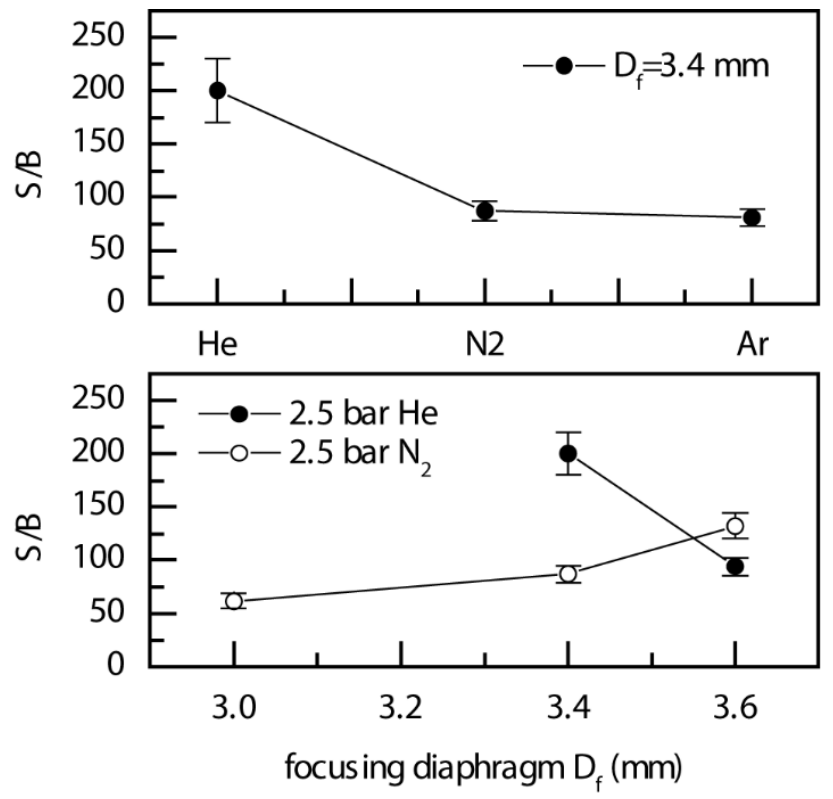

Figure S3. The dependence of the S/B ratio on (b) the type of the carrier gas and (c) the diameter of the focusing diaphragm $D_{\mathrm{f}}$ for tryptophan aerosol particles recorded at hv=8 eV.

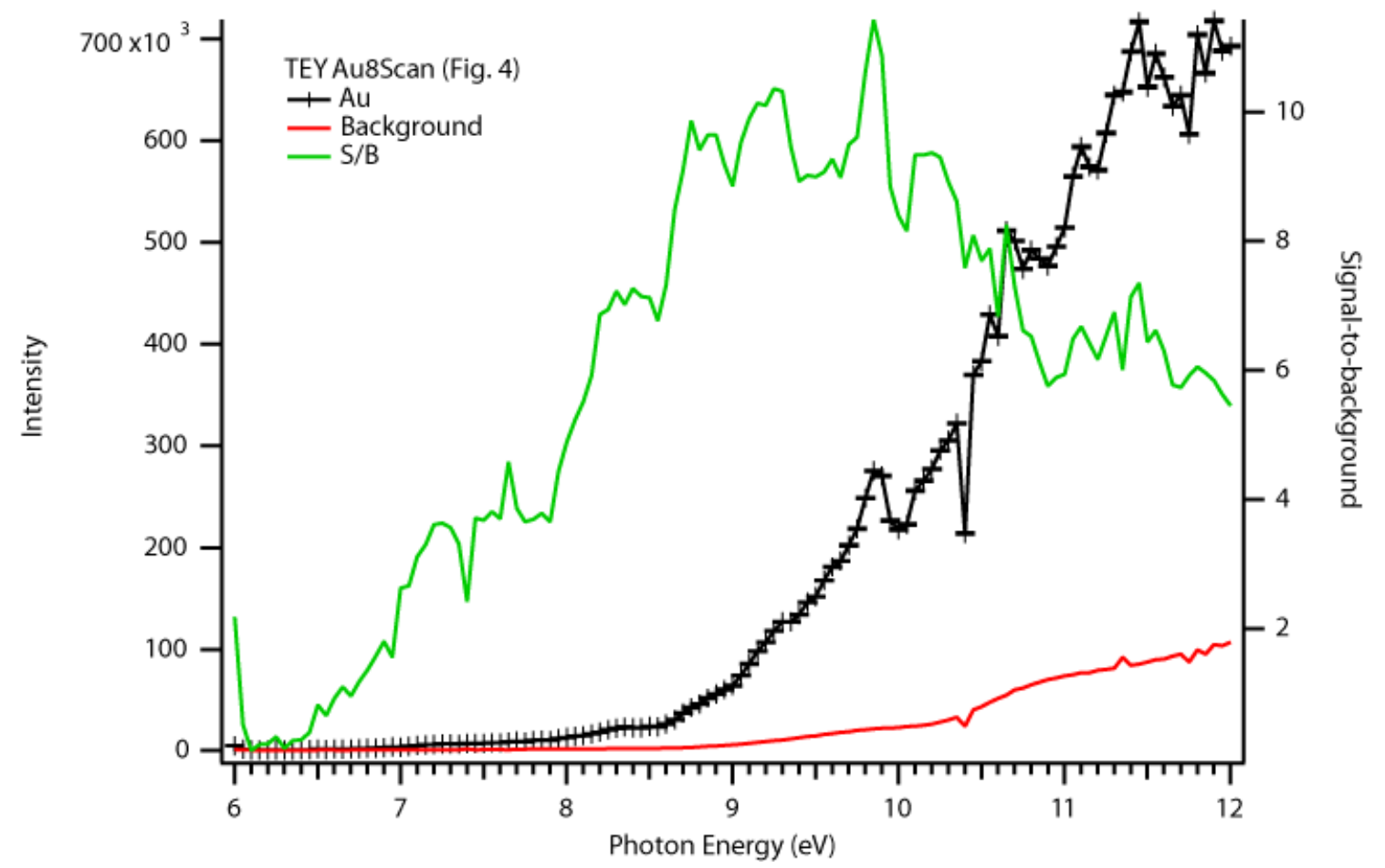

Figure S4. The total electron yields for the AuNP-0 sample (black line) and the background (red), as well as the dependence of the ratio between the two signals (green) on the phonon energy. 


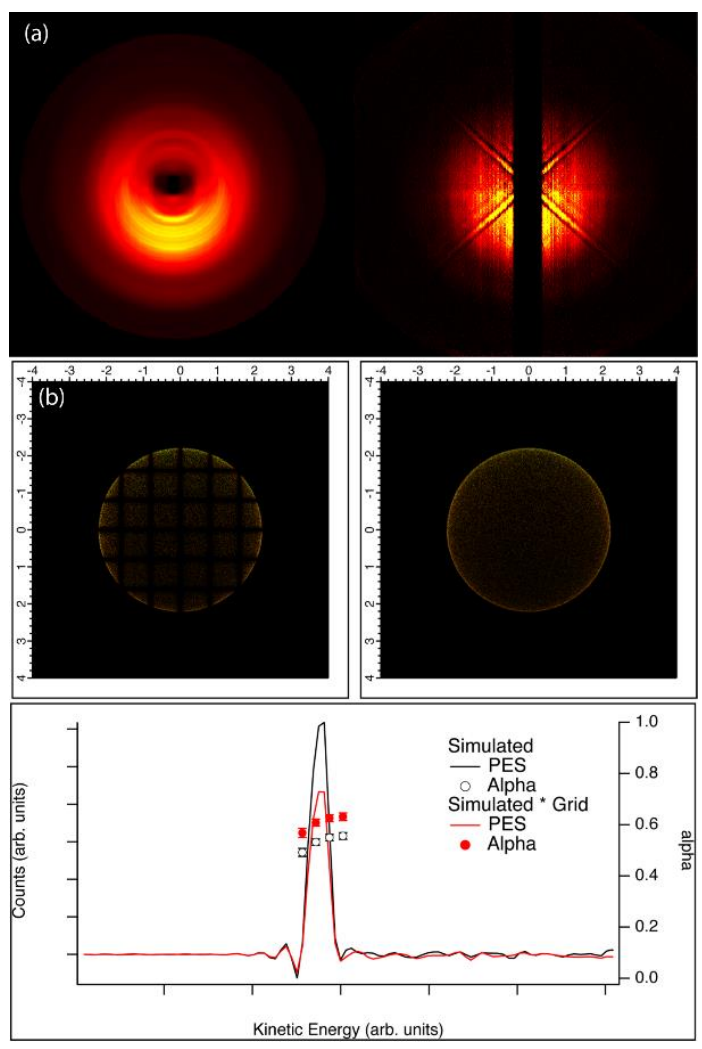

Figure S5. (a) The photoelectron images of the AuNP-0 sample recorded at hv=10.2 eV reconstructed by modified pBasex (left) and BASEX (right) algorithms. (b) A simulation showing the effects of a coarse grid pattern observed in the photoelectron images on the VMI parameters obtained by the modified pBasex algorithm.

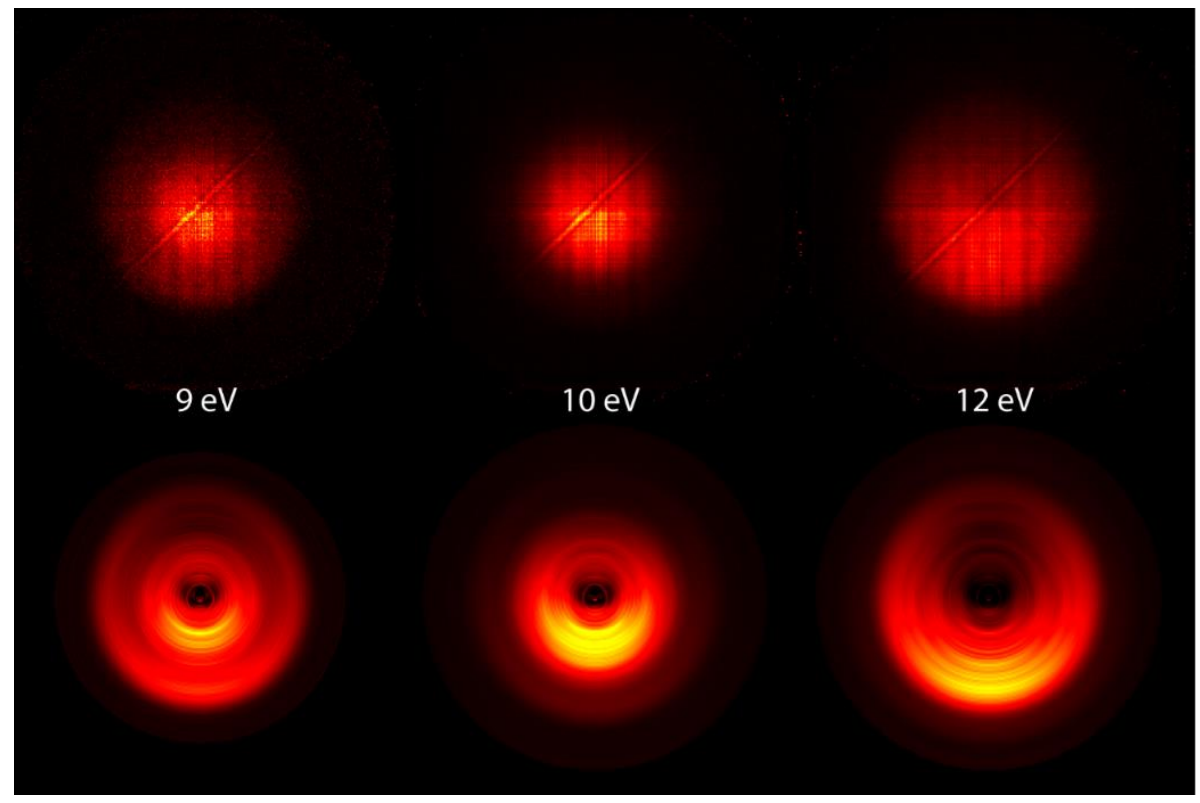

Figure S6. The background-corrected raw and pBasex reconstructed photoelectron image of AuNP-0 recorded at $h v=9 \mathrm{eV}, 10 \mathrm{eV}$, and $12 \mathrm{eV}$. 


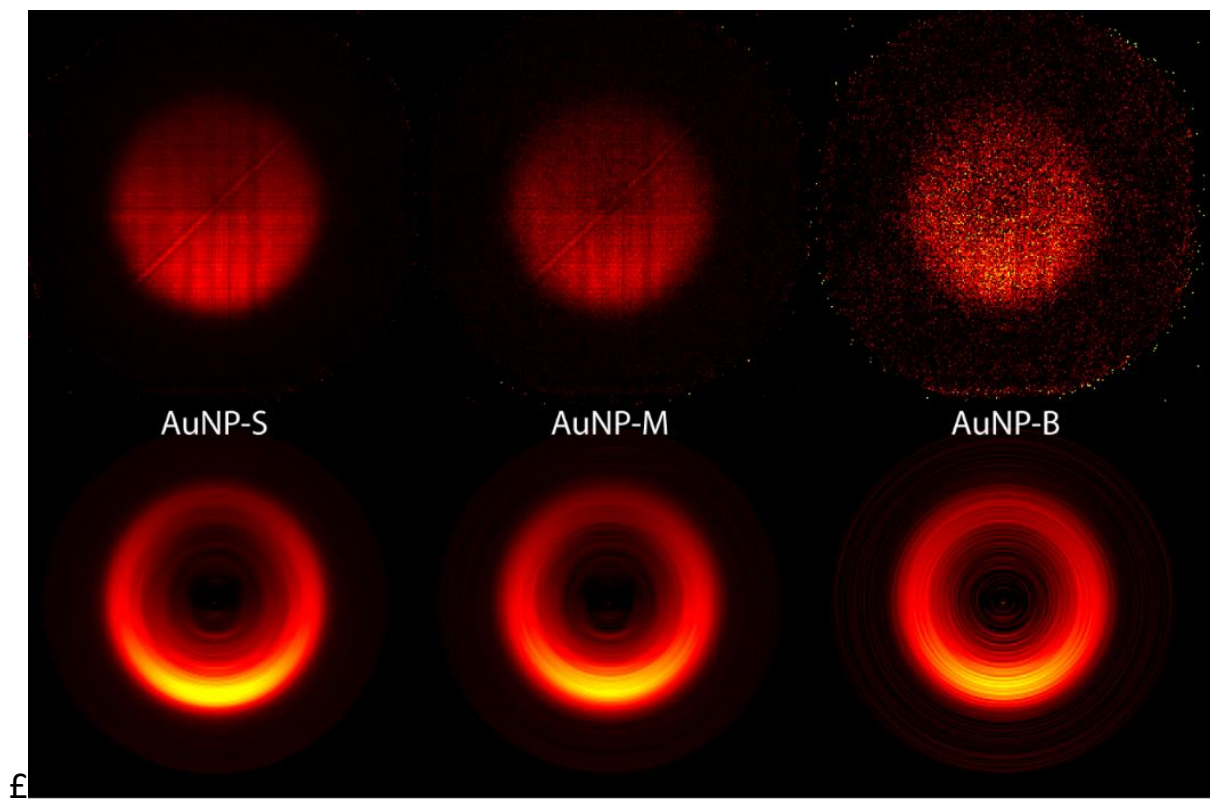

Figure S7. The background-corrected raw and pBasex reconstructed photoelectron image of the AuNP-S, AuNP-M, and AuNP-B sample recorded at $h v=12 \mathrm{eV}$.

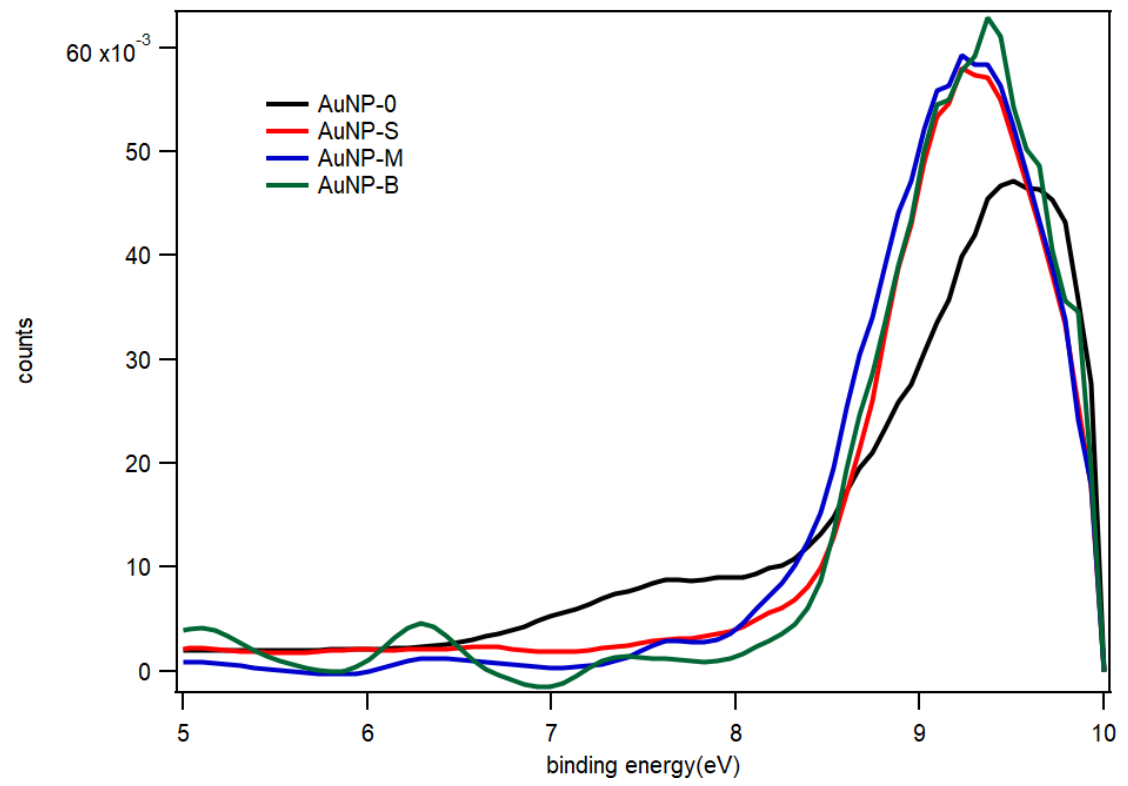

Figure S8. The photoemission spectra of the AuNP-0, AuNP-S, AuNP-M, and AuNP-B sample recorded at $h v=10 \mathrm{eV}$. 